\title{
Correction to: Case-management protocol for bloody diarrhea as a model to reduce the clinical impact of Shiga toxin-producing Escherichia coli infections. Experience from Southern Italy
}

\author{
Daniela Loconsole $^{1}$ (D) $\cdot$ Mario Giordano ${ }^{2}$ (D) $\cdot$ Nicola Laforgia $^{3}$ (D) $\cdot$ Diletta Torres $^{2} \cdot$ Luisa Santangelo $^{2}$. \\ Vincenza Carbone ${ }^{2}$. Antonio Parisi ${ }^{4}$ (D) Michele Quarto $^{1} \cdot$ Gaia Scavia $^{5}$ (D) $\cdot$ Maria Chironna ${ }^{1}$ (D) Bloody Diarrhea \\ Apulia Working Group
}

Published online: 19 December 2019

(C) Springer-Verlag GmbH Germany, part of Springer Nature 2019

\section{Correction to: European Journal of Clinical Microbiology \& Infectious Diseases} https://doi.org/10.1007/s10096-019-03755-0

In the originally published article, the name of the $8^{\text {th }}$ author Michele Quarto was inadvertently omitted during typesetting. Author's name is now correctly captured above.

Also, the collaborators of the institutional group "Bloody Diarrhea Apulia Working Group" are given below:

Luigi Nigri, Viviana Bruno, Simona Baldacci, Francesca Centrone, Anna Lisa De Robertis, Anna Morea, Daniele Casulli, Marisa Accogli, Serafina Rutigliano and Onofrio Mongelli

The original article has been corrected.

The online version of the original article can be found at https://doi.org/ 10.1007/s10096-019-03755-0

Maria Chironna

maria.chironna@uniba.it

Daniela Loconsole

daniela.loconsole@uniba.it

Mario Giordano

mariogiordanobari@fastwebnet.it

Nicola Laforgia

nicola.laforgia@uniba.it

Diletta Torres

dilettatorres@libero.it

Luisa Santangelo

luisa.santangelo@hotmail.it

Vincenza Carbone

enzacarbone2009@libero.it

Antonio Parisi

antonio.parisi@izspb.it
Michele Quarto

miche.quarto@uniba.it

Gaia Scavia

gaia.scavia@iss.it

1 Department of Biomedical Sciences and Human Oncology, Hygiene Unit, University of Bari Aldo Moro, P.zza G. Cesare 11, 70124 Bari, Italy

2 Pediatric Nephrology and Dialysis Unit, Pediatric Hospital "Giovanni XXIII", Bari, Italy

3 Department of Biomedical Sciences and Human Oncology, Neonatal Intensive Care Unit, University of Bari Aldo Moro, Bari, Italy

4 Istituto Zooprofilattico Sperimentale della Puglia e della Basilicata, Foggia, Italy

5 Food Safety, Nutrition and Veterinary Public Health Department, Istituto Superiore di Sanità, Rome, Italy 